\title{
Analysis of Pilot-Assisted Channel Estimators for OFDM Systems With Transmit Diversity
}

\author{
Dongxu Shen, Member IEEE, Zhifeng Diao, Kai-Kit Wong, Member IEEE, and Victor O. K. Li, Fellow IEEE
}

\begin{abstract}
In this paper, we analyze the performance of pilot-assisted least square (LS) and minimum mean squared error (MMSE) channel estimators for orthogonal frequency division multiplexing (OFDM) systems with transmit antenna diversity. We first provide a design of orthogonal pilot sequences to simplify the estimators. We then analyze the mean squared error (MSE) performance, and study the leakage effect. When a channel tap is not sample-spaced, our analysis shows that the power of the channel tap will leak to not only other taps of the same antenna, but also taps belonging to other antennas. The leakage across antennas is mainly determined by the phase separation between pilot sequences, which is further related to the ratio between the number of pilots and number of antennas. We demonstrate that the MSE performance can be improved if more pilots are used, or fewer channels are estimated simultaneously.
\end{abstract}

Index Terms-Channel estimation, leakage effect, OFDM, pilot sequence, space time coding.

\section{INTRODUCTION}

$\mathbf{R}$ ECENTLY, there is growing interest to apply transmit diversity techniques such as space-time coding (STC) to OFDM systems for the mitigation of fading [1]. The implementation of transmit diversity requires the availability of channel state information at the receiver. Therefore, reliable channel estimation is a prerequisite for the realization of space-time coded OFDM [1].

There has been much research on channel estimation for OFDM systems where the transmitter has a single antenna element [2]-[10]. All the above approaches are not directly applicable to systems with transmit diversity, because the interference from multiple transmit antennas has to be resolved at the receive antenna.

Channel estimation with transmit diversity has been studied in [11]-[15]. In [11], Y. Li et al. proposed an algorithm applicable to transmit diversity. Since the algorithm in [11] involves complex matrix inversion, a simplification of [11] was later provided in [12]. Based on the algorithms in [11], [12], H. Minn et al. [14] investigated a reduced-complexity channel estimation algorithm by assuming that two neighboring subcarriers have the same channel gain, thereby reducing the computational complexity by half. The schemes in [11]-[14] all

Manuscript received on Jan 18 2005; revised April 3, 2006. This research is supported in part by the Research Grants Council of Hong Kong under Grant HKU 7047/00E.

D. Shen, Z. Diao, and V. O. K. Li are with the Department of Electrical and Electronic Engineering, University of Hong Kong, Hong Kong (e-mail: dxshen@eee.hku.hk; zfdiao@eee.hku.hk; vli@eee.hku.hk).

K.-K. Wong is with the Department of Engineering, University of Hull, Kingston-upon-Hull HU6 7RX, U.K. (e-mail: k.wong@hull.ac.uk).

Digital Object Identifier 10.1109/TBC.2005.854172 adopt a sample-spaced approach by assuming that channel taps are sample-spaced, which causes power leakage among channel taps [2], [14]. The leakage effect for the sample-spaced approaches is not thoroughly studied. The leakage effect can be overcome by the nonsample-spaced approach [15], based on the estimation of tap delays. However, it is much more complex than the sample-spaced approaches. Thus, the sample-spaced approach is attractive in its simplicity, but a thorough investigation on the leakage effect is needed. Further, in all of these efforts, a whole OFDM symbol is devoted to channel estimation, and the rich correlation existing across neighboring subcarriers is unexploited.

In this paper, we first develop pilot-assisted least square (LS) and minimum mean squared error (MMSE) channel estimators for OFDM systems with transmit antenna diversity, based on the sample-spaced approach. By using only a portion of OFDM subcarriers as pilots, bandwidth efficiency is improved since the remaining subcarriers can be used for carrying data. We then give a design of orthogonal pilot sequences, which reduces the computational complexity, and lowers the mean squared error (MSE) [11]. The approach in [12] uses all subcarriers as pilots in an OFDM symbol for channel estimation, which could be wasteful of resource. [13] distributes pilots in several OFDM symbols, which introduces processing delay and complexity. In this work, pilot sequence is inserted in one OFDM symbol. We also analyze the performance of the estimators, especially under the influence of the leakage effect. Our analysis shows that when the channel taps are nonsample-spaced, tap power will not only leak to the taps of the same antenna, but also leak to the channel taps of other antennas. Given the tap delay, the amount of cross-antenna leakage is mainly influenced by the phase separation between the pilot sequences, which is determined by the ratio between the number of pilots and the number of transmit antennas. Thus, performance can be improved by either using more pilots, or estimating fewer channels simultaneously.

This paper is organized as follows. In Section II, the system and the channel model are described. In Section III, the LS and MMSE channel estimators are derived. Design of orthogonal pilot sequences is provided in Section IV. In Section V, we analyze the performance of the simplified estimators. Simulation results are presented in Section VI. Conclusion is given in Section VII.

\section{System DesCRIPTION AND CHANNEl Model}

\section{A. System Description}

Without loss of generality, we consider an OFDM-based transmit diversity system with $A$ transmit antenna elements 


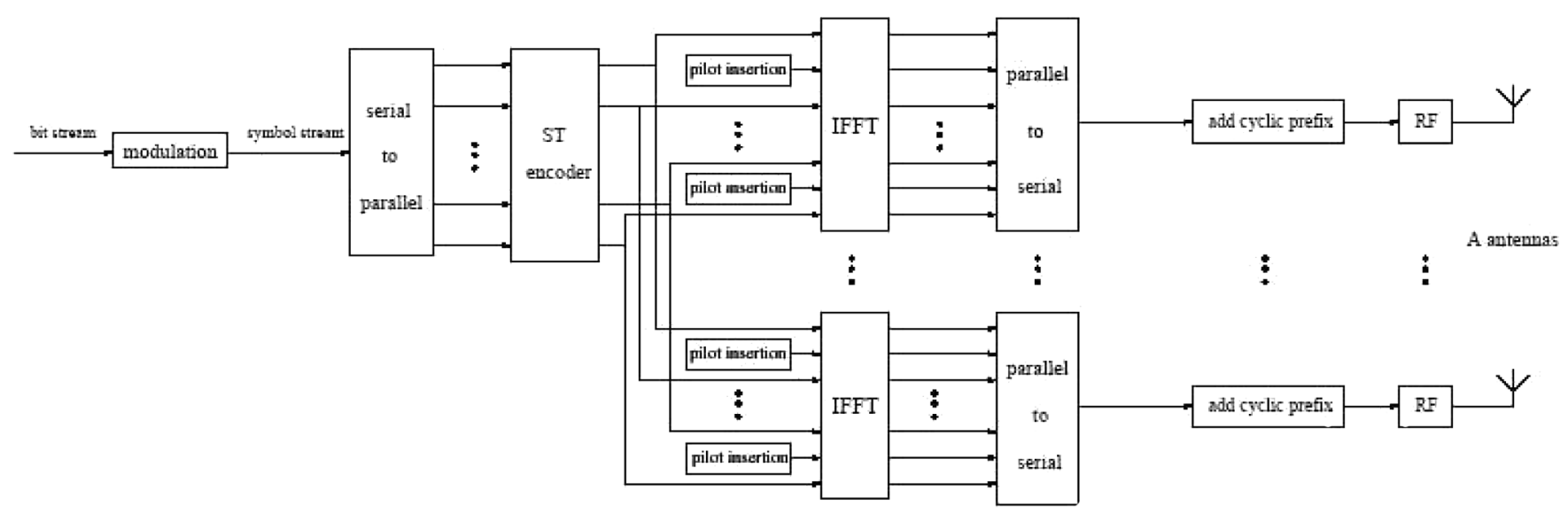

(a)

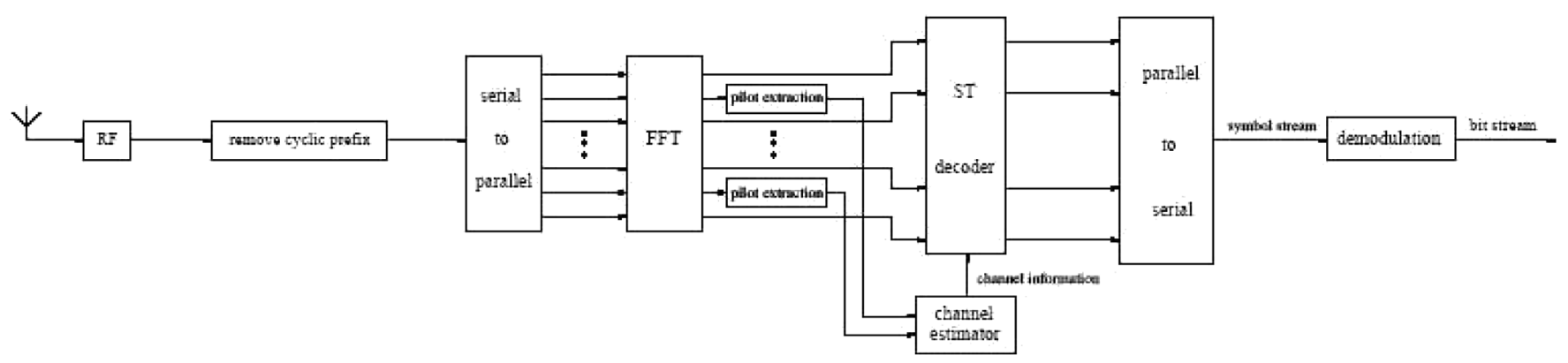

(b)

Fig. 1. Schematic of a space-time coded OFDM system with pilot-assisted channel estimation. (a) transmitter; (b) receiver.

and one receive antenna. The channel estimation methods are directly applicable to MIMO systems. We also assume perfect synchronization. An illustration of the system is provided in Fig. 1.

\section{B. Channel Model}

We call the channel between the $i$ th transmit antenna and the receive antenna the $i$ th channel in the system. With $A$ transmit antennas, there are a total of $A$ channels. We assume all channels are independent.

The baseband representation of the time domain channel impulse response of the $i$ th channel is characterized as

$$
\Gamma_{i}(t, \tau)=\sum_{l=0}^{L-1} \alpha_{i, l}(t) \delta\left(\tau-\tau_{i, l} \Delta t\right)
$$

where $\alpha_{i, l}(t)$ is the complex gain of path $l, \tau_{i, l}$ is the corresponding path delay, $L$ is the number of paths, $\delta(\tau)$ is the Dirac delta function, and $\Delta t$ is the sampling interval of the OFDM system. We assume that $\alpha_{i, l}(t)$ is a wide-sense stationary (WSS) complex Gaussian process with uncorrelated scattering (US) between different paths. Further, the channel is assumed to be constant during an OFDM symbol, and the average channel power gain is normalized to unity, i.e., $\sum_{l=0}^{L-1} E\left\{\alpha_{i, l}^{2}(t)\right\}=1$. Here $E\{\cdot\}$ denotes the expectation operation. We also assume the maximal tap delay is always shorter than the cyclic prefix.
Corresponding to (1), the frequency domain channel response can be expressed as

$$
H_{i}(n, k)=\frac{1}{\sqrt{N_{c}}} \sum_{l=0}^{L-1} \alpha_{i, l}\left(n T_{s}\right) W^{k \tau_{i, l}}
$$

where $n$ is the time index for an OFDM symbol, $k$ is the subcarrier index, $T_{s}$ is the duration of an OFDM symbol, and $W=e^{-j 2 \pi / N_{c}}$ with $N_{c}$ being the number of OFDM subcarriers. Since pilots are inserted in the same OFDM symbol, we drop the index $n$ for conciseness. Generally speaking, $\tau_{i, l}$ 's are not integers.

Let $\mathbf{h}_{i}=\left[\begin{array}{llll}\alpha_{i, 1} & \alpha_{i, 2} & \cdots & \alpha_{i, L}\end{array}\right]^{T}$ be the channel vector for antenna $i$. The time domain channel vector for all antennas is defined as

$$
\mathbf{h} \triangleq\left[\begin{array}{llll}
\mathbf{h}_{1}^{T} & \mathbf{h}_{2}^{T} & \cdots & \mathbf{h}_{A}^{T}
\end{array}\right]^{T} \in \mathbb{C}^{A L}
$$

\section{PILOT-Assisted LS AND MMSE CHANNEL ESTIMATORS}

\section{A. Problem Formulation}

We assume that all pilots are placed in the same OFDM symbol in an STC block. Suppose that $K$ subcarriers are used as pilots, and let $\mathbb{X}$ be the set of subcarrier indices for pilots. The received signal on the pilot subcarrier $x_{k} \in \mathbb{X}$ is given by

$$
y\left(x_{k}\right)=\sum_{i=1}^{A} H_{i}\left(x_{k}\right) s_{i}\left(x_{k}\right)+n\left(x_{k}\right), k=1, \ldots, K,
$$


where $H_{i}\left(x_{k}\right)$ is the frequency domain channel response on pilot subcarrier $x_{k}$ for transmit antenna $i, s_{i}\left(x_{k}\right)$ is the pilot symbol on subcarrier $x_{k}$ for antenna $i$, and $n\left(x_{k}\right)$ is a zero- mean complex random variable for additive white Gaussian noise (AWGN) with variance $\sigma_{0}^{2}$. We call the sequence $\left\{s_{i}\left(x_{1}\right), s_{i}\left(x_{2}\right), \cdots, s_{i}\left(x_{K}\right)\right\}$ the pilot sequence for antenna $i$.

Let $\mathbf{S}_{i}=\operatorname{diag}\left\{s_{i}\left(x_{1}\right), s_{i}\left(x_{2}\right), \cdots, s_{i}\left(x_{K}\right)\right\} \in \mathbb{C}^{K \times K}$, and $\mathbf{S}=\left[\begin{array}{lll}\mathbf{S}_{1} & \mathbf{S}_{2} \cdots & \mathbf{S}_{A}\end{array}\right] \in \mathbb{C}^{K \times A K}$. Likewise, let $\mathbf{Y}=\left[\begin{array}{llll}y\left(x_{1}\right) & y\left(x_{2}\right) & \cdots & y\left(x_{K}\right)\end{array}\right]^{T} \in \mathbb{C}^{K}$, and $\mathbf{n}=$ $\left[n\left(x_{1}\right) n\left(x_{2}\right) \cdots n\left(x_{K}\right)\right]^{T} \in \mathbb{C}^{K}$. The expressions in (4) can be reformulated into matrix form as

$$
\mathbf{Y}=\mathbf{S H}+\mathbf{n},
$$

where $\mathbf{H} \triangleq\left[\begin{array}{llll}\mathbf{H}_{1}^{T} & \mathbf{H}_{2}^{T} & \cdots & \mathbf{H}_{A}^{T}\end{array}\right]^{T} \in \mathbb{C}^{A K}$, with $\mathbf{H}_{i}=$ $\left[H_{i}\left(x_{1}\right) H_{i}\left(x_{2}\right) \cdots H_{i}\left(x_{K}\right)\right]^{T} \in \mathbb{C}^{K}$. Here $\mathbf{H}=\mathbf{W}_{N S} \mathbf{h}$, and

$$
\mathbf{W}_{N S}=\left[\begin{array}{cccc}
\mathbf{W}_{N S, 1} & \mathbf{0} & \cdots & \mathbf{0} \\
\mathbf{0} & \mathbf{W}_{N S, 2} & \cdots & \mathbf{0} \\
\vdots & \vdots & \ddots & \vdots \\
\mathbf{0} & \mathbf{0} & \cdots & \mathbf{W}_{N S, A}
\end{array}\right] \in \mathbb{C}^{A K \times A L}
$$

where the $(i, j)$ th element of $\mathbf{W}_{N S, i} \in \mathbb{C}^{K \times L}$ is given by $\left(1 / \sqrt{N_{c}}\right) W^{x_{i} \tau_{i, j}}$. Equation (5) is finally re-written as

$$
\begin{aligned}
\mathbf{Y} & =\mathbf{S W}_{N S} \mathbf{h}+\mathbf{n} \\
& =\mathbf{P}_{N S} \mathbf{h}+\mathbf{n},
\end{aligned}
$$

where $\mathbf{P}_{N S}=\mathbf{S} \mathbf{W}_{N S}$, and $\mathbf{P}_{N S} \in \mathbb{C}^{K \times A L}$.

\section{B. Sample-Spaced LS and MMSE Channel Estimators}

For a nonsample-spaced approach, $\tau_{i, j}$ 's $i=1, \ldots, A$, $j=1, \ldots L$ can either be assumed random [5], or be estimated [8], [15], both of which involve much complexity. For a sample-spaced approach, $\tau_{i, j}$ 's are assumed to be integers, which greatly simplifies the estimator. In this paper, we also adopt the sample-spaced approach. Thus, in (8), $\mathbf{P}_{N S}$ is substituted by $\mathbf{P}=\mathbf{S W}$, where

$$
\mathbf{W}=\left[\begin{array}{cccc}
\mathbf{W}_{1} & \mathbf{0} & \cdots & \mathbf{0} \\
\mathbf{0} & \mathbf{W}_{2} & \cdots & \mathbf{0} \\
\vdots & \vdots & \ddots & \vdots \\
\mathbf{0} & \mathbf{0} & \cdots & \mathbf{W}_{A}
\end{array}\right] \in \mathbb{C}^{A K \times A L^{\prime}}
$$

In (9), the $(i, j)$ th element of $\mathbf{W}_{i}$ is $\left(1 / \sqrt{N_{c}}\right) W^{x_{i}} \tau_{i, j}$, and $\mathbf{W}_{i} \in \mathbb{C}^{K \times L^{\prime}}$ where $L^{\prime}$ is the number of estimated channel taps. The selection of $L^{\prime}$ is discussed later. Here we assume $L^{\prime} \geq L$. Then $\mathbf{P}$ is of dimension $K \times A L^{\prime}$.

Since $\mathbf{P}$ is linear with respect to $\mathbf{h}$, and $\mathbf{n}$ is Gaussian, a sample-spaced $\mathrm{LS}^{1}$ channel estimator can be derived as [16]

$$
\widehat{\mathbf{h}}_{L S}=\left(\mathbf{P}^{H} \mathbf{P}\right)^{-1} \mathbf{P}^{H} \mathbf{Y}
$$

where $H$ is the Hermitian transpose operator. Let $\mathbf{Q}=\mathbf{P}^{H} \mathbf{P} \in$ $\mathbb{C}^{A L^{\prime} \times A L^{\prime}}$. For $\mathbf{Q}$ to be invertible, it is necessary to have $K \geq$ $A L^{\prime}$. Then the estimator can be re-expressed as

$$
\widehat{\mathbf{h}}_{L S}=\mathbf{Q}^{-1} \mathbf{P}^{H} \mathbf{Y} \text {. }
$$

${ }^{1}$ For this channel estimation problem, the LS estimator is also the maximum likelihood (ML) estimator.
An MMSE estimator [16] is given by

$$
\widehat{\mathbf{h}}_{M M S E}=\mathbf{R}_{\mathbf{h Y}} \mathbf{R}_{\mathbf{Y Y}}^{-1} \mathbf{Y},
$$

where $\mathbf{R}_{\mathbf{h Y}}=E\left\{\mathbf{h} \mathbf{Y}^{H}\right\}=\mathbf{R}_{\mathbf{h h}} \mathbf{P}_{N S}^{H}$, and $\mathbf{R}_{\mathbf{Y Y}}=$ $E\left\{\mathbf{Y} \mathbf{Y}^{H}\right\}=\left(\mathbf{P}_{N S} \mathbf{R}_{\mathbf{h h}} \mathbf{P}_{N S}^{H}+\sigma^{2} \mathbf{I}\right)$. Since $\mathbf{P}_{N S}$ is unknown, we use $\mathbf{P}$ instead. Then the MMSE estimator becomes

$$
\begin{aligned}
\widehat{\mathbf{h}}_{M M S E} & =\mathbf{R}_{\mathbf{h h}}\left(\mathbf{R}_{\mathbf{h h}}+\sigma^{2}\left(\mathbf{P}^{H} \mathbf{P}\right)^{-1}\right)^{-1}\left(\mathbf{P}^{H} \mathbf{P}\right)^{-1} \mathbf{P}^{H} \mathbf{Y} \\
& =\mathbf{R}_{\mathbf{h h}}\left(\mathbf{R}_{\mathbf{h h}}+\sigma^{2} \mathbf{Q}^{-1}\right)^{-1} \widehat{\mathbf{h}}_{L S} .
\end{aligned}
$$

$\mathbf{R}_{\mathbf{h h}}$ is the autocorrelation matrix of all channels. Since channels are independent,

$$
\mathbf{R}_{\mathbf{h h}}=\left[\begin{array}{cccc}
\mathbf{R}_{1} & \mathbf{0} & \cdots & \mathbf{0} \\
\mathbf{0} & \mathbf{R}_{2} & \cdots & \mathbf{0} \\
\vdots & \vdots & \ddots & \vdots \\
\mathbf{0} & \mathbf{0} & \cdots & \mathbf{R}_{A}
\end{array}\right]
$$

where $\mathbf{R}_{i}=E\left\{\mathbf{h}_{i} \mathbf{h}_{i}^{H}\right\}$. With uncorrelated channel taps, $\mathbf{R}_{i}=\operatorname{diag}\left\{\alpha_{i, 0}^{2}, \alpha_{i, 1}^{2}, \ldots, \alpha_{i, L-1}^{2}, 0, \ldots, 0\right\}$. Here $\mathbf{R}_{i}$ is padded with 0 's in order to have dimension $L^{\prime}$.

In practice, $\mathbf{R}_{\mathbf{h h}}$ is unknown and has to be estimated, which complicates the estimator. According to [6], robust channel estimation can be achieved by assuming uniform power distribution on channel taps. Thus we set $\widetilde{\mathbf{R}}_{i}=$ $\operatorname{diag}\left\{1 / L^{\prime}, 1 / L^{\prime}, \ldots, 1 / L^{\prime}\right\} \in \mathbf{R}^{L^{\prime} \times L^{\prime}}, i=1, \ldots, A$, and

$$
\widetilde{\mathbf{R}}_{\mathbf{h h}}=\left[\begin{array}{cccc}
\widetilde{\mathbf{R}}_{1} & \mathbf{0} & \cdots & \mathbf{0} \\
\mathbf{0} & \widetilde{\mathbf{R}}_{2} & \cdots & \mathbf{0} \\
\vdots & \vdots & \ddots & \vdots \\
\mathbf{0} & \mathbf{0} & \cdots & \widetilde{\mathbf{R}}_{A}
\end{array}\right]
$$

As a consequence, the MMSE estimator becomes

$$
\widetilde{\mathbf{h}}_{M M S E}=\widetilde{\mathbf{R}}_{\mathbf{h h}}\left(\widetilde{\mathbf{R}}_{\mathbf{h h}}+\sigma^{2} \mathbf{Q}^{-1}\right)^{-1} \widehat{\mathbf{h}}_{L S} .
$$

\section{Design of Orthogonal Pilot Sequences}

The estimators in Section III can be simplified by employing orthogonal pilot sequences. Further, according to [12], optimal MSE performance can be achieved with orthogonal pilot sequences.

\section{A. Generation of Orthogonal Pilot Sequences}

An approach for orthogonal sequence design is given in [12], but it is not applicable to pilot-assisted cases. The details of our approach are given in the Appendix. Here we briefly outline the sequence generation procedure:

1) The modulation for the pilot sequence of Antenna $i$ has constant modulus, i.e., $\left|s_{i}(k)\right|^{2}=E_{0}, \forall k$, where $E_{0}$ is the pilot power;

2) The ratio between the number of subcarriers in an OFDM symbol and the number of pilot subcarriers is an integer, i.e., $N_{c} \bmod K \equiv 0$;

3) The pilot locations are equally spaced, i.e., $x_{i+1}-x_{i}=$ $x_{j+1}-x_{j}, 1 \leq i, j \leq K-1$

4) Sequences for Antenna $i, 2 \leq i \leq A$ are generated from the pilot sequence of Antenna 1 as

$s_{i}(k)=s_{1}(k) e^{-j 2 \pi U(i-1) x_{k} / N_{c}}, \quad 1 \leq k \leq K$

where $U$ is defined as the phase separation between pilot sequences. We require $A U \leq K$ and $U \geq L^{\prime}$. 


\section{B. Simplified Estimators}

Using orthogonal pilot sequences, the inverse of $\mathbf{Q}$ (see details in the Appendix) is simply

$$
\mathrm{Q}^{-1}=\frac{N_{c}}{K E_{0}} \mathbf{I}_{A L^{\prime}} .
$$

Here we use $\mathbf{I}_{M}$ to denote an identity matrix of dimension $M$, and we assume all pilot sequences have the same pilot power of $E_{0}$. From (11), the LS estimator becomes

$$
\widehat{\mathbf{h}}_{L S}=\frac{N_{c}}{K E_{0}} \mathbf{P}^{H} \mathbf{Y} .
$$

From (13), the MMSE estimator becomes

$$
\widehat{\mathbf{h}}_{M M S E}=\mathbf{D} \widehat{\mathbf{h}}_{L S},
$$

where $\mathrm{D}$ is a diagonal matrix and its $\left[(i-1) L^{\prime}+(l+\right.$ 1)] thelement is

$$
\begin{cases}\frac{\alpha_{i, l}^{2}}{\alpha_{i, l}^{2}+\frac{N_{c} \sigma^{2}}{K E_{0}}}, & 0 \leq l \leq L-1 \\ 0, & L \leq l \leq L^{\prime}-1 .\end{cases}
$$

The robust MMSE estimator is simplified from (15) as

$$
\widetilde{\mathbf{h}}_{M M S E}=d_{0} \widehat{\mathbf{h}}_{L S},
$$

where $d_{0}=1 /\left(1+L^{\prime} / \gamma\right)$, and $\gamma=\left(K E_{0} / N_{c} \sigma^{2}\right)$. In practice, the ratio $E_{0} / \sigma^{2}$ is also unknown. Therefore, it is suggested in [6] that $E_{0} / \sigma^{2}$ should be selected as a high signal-to-noise ratio (SNR) value. In that case, $d_{0} \approx 1$ and the robust MMSE estimator is equivalent to the LS estimator.

\section{AnAlysis of Estimator Performance}

In this section, we analyze the MSE performance of the estimators when orthogonal pilot sequences are adopted.

\section{A. MSE Performance}

1) MSE of LS Estimator: Substituting (8) into (10), we have

$$
\begin{aligned}
\widehat{\mathbf{h}}_{L S} & =\left(\mathbf{P}^{H} \mathbf{P}\right)^{-1} \mathbf{P}^{H}\left(\mathbf{P}_{N S} \mathbf{h}+\mathbf{n}\right) \\
& =\left(\mathbf{P}^{H} \mathbf{P}\right)^{-1} \mathbf{P}^{H} \mathbf{P}_{N S} \mathbf{h}+\left(\mathbf{P}^{H} \mathbf{P}\right)^{-1} \mathbf{P}^{H} \mathbf{n} .
\end{aligned}
$$

It is obvious that the LS estimator is unbiased only when $\mathbf{P}_{N S}=$ $\mathbf{P}$, i.e., the channel is sample-spaced.

Let $\widehat{\mathbf{H}}_{L S}=\mathbf{W} \widehat{\mathbf{h}}_{L S}$ be the frequency domain channel estimate. The MSE of the LS estimator can be derived as

$$
\begin{aligned}
M S E_{L S}= & E\left\{(\hat{\mathbf{H}})^{H}\left(\hat{\mathbf{H}}_{L S}-\mathbf{H}\right)\right\} \\
= & E\left\{(\mathbf{B h})^{H}(\mathbf{B h})\right\} \\
& +E\left\{\left(\mathbf{W} \mathbf{Q}^{-1} \mathbf{P}^{H} \mathbf{n}\right)^{H}\left(\mathbf{W Q}^{-1} \mathbf{P}^{H} \mathbf{n}\right)\right\},
\end{aligned}
$$

where

$$
\mathbf{B}=\mathbf{W}\left(\mathbf{P}^{H} \mathbf{P}\right)^{-1} \mathbf{P}^{H} \mathbf{P}_{N S}-\mathbf{W}_{N S} \in \mathbb{C}^{A N_{c} \times A L} .
$$

Matrix B characterizes the influence of leakage on MSE. Obviously, $\mathbf{B}$ is a zero matrix when channels are sample-spaced; when channels are nonsample-spaced, $\mathbf{B}$ is a nonzero matrix.
Thus, the MSE has two parts: the noise part, and the leakage part. Here we first analyze the noise part, and defer the investigation on the leakage effect to Section V-B.

The noise part of MSE can be re-expressed as

$$
\begin{aligned}
M S E_{L S, \text { noise }} & =E\left\{\operatorname{tr}\left(\left(\mathbf{W Q}^{-1} \mathbf{P}^{H} \mathbf{n}\right)\left(\mathbf{W Q}^{-1} \mathbf{P}^{H} \mathbf{n}\right)^{H}\right)\right\} \\
& =\operatorname{tr}\left(\sigma_{0}^{2} \mathbf{W} \mathbf{Q}^{-1} \mathbf{W}^{H}\right) .
\end{aligned}
$$

When orthogonal pilot sequences are employed, the MSE is

$$
\begin{aligned}
M S E_{L S, \text { noise }} & =\frac{N_{c} \sigma_{0}^{2}}{K E_{0}} \cdot \frac{L^{\prime}}{N_{c}} \cdot A N_{c} \\
& =\frac{A L^{\prime} N_{c} \sigma_{0}^{2}}{K E_{0}} .
\end{aligned}
$$

The MSE on subcarrier $k$ of antenna $i$ is given by the corresponding diagonal element of $\sigma_{0}^{2} \mathbf{W} \mathbf{Q}^{-1} \mathbf{W}^{H}$, which is

$$
M S E_{L S, n o i s e}(i, k)=\frac{L^{\prime} \sigma_{0}^{2}}{K E_{0}}
$$

Thus, the noise part of MSE is identical for all subcarriers.

2) MMSE Estimator: With orthogonal pilot sequences, the MMSE estimators, given in (18) and (19), are weighted LS estimators. Thus, the MSE analysis is similar to that for the LS estimator. From (18), the MSE of the MMSE estimator can be derived as

$$
\begin{aligned}
M S E_{M M S E}= & E\left\{\left(\widehat{\mathbf{H}}_{M M S E}-\mathbf{H}\right)^{H}\left(\widehat{\mathbf{H}}_{M M S E}-\mathbf{H}\right)\right\} \\
= & E\left\{\left(\mathbf{B}^{\prime} \mathbf{h}\right)^{H}\left(\mathbf{B}^{\prime} \mathbf{h}\right)\right\} \\
& +E\left\{\left(\mathbf{W D Q}^{-1} \mathbf{P}^{H} \mathbf{n}\right)^{H}\left(\mathbf{W D Q}^{-1} \mathbf{P}^{H} \mathbf{n}\right)\right\},
\end{aligned}
$$

where $\mathbf{B}^{\prime}=\mathbf{W D}\left(\mathbf{P}^{H} \mathbf{P}\right)^{-1} \mathbf{P}^{H} \mathbf{P}_{N S}-\mathbf{W}_{N S} \in \mathbb{C}^{A N_{c} \times A L}$. The MSE caused by noise is given by

$$
M S E_{M M S E, \text { noise }}=\frac{L^{\prime} \sigma_{0}^{2}}{K E_{0}} \sum_{j=1}^{A N_{c}} d_{j}^{2}
$$

where $d_{j}$ is the $j$ th element of $\mathbf{D}$.

For the robust MMSE estimator, the noise part of MSE is given by

$$
M S E_{M M S E, \text { noise, robust }}=\frac{A N_{c} d_{0} L^{\prime} \sigma_{0}^{2}}{K E_{0}} .
$$

3) Discussion: When the channel is sample-spaced, the channel estimation quality for the above estimators is solely given by (23), (26) and (27). In that case, if $A, L^{\prime}, N_{c}, \sigma_{0}^{2}$ and $d_{j}$ 's are fixed, we can observe from the above equations that the channel MSE is only determined by $K E_{0}$, which happens to be the total energy on pilot subcarriers. Thus, there is a tradeoff between the number of pilots and pilot energy. In other words, channel MSE can be maintained by reducing $K$ and proportionally increasing $E_{0}$. 
On the other hand, if only $A$ and $K$ are allowed to vary, and other parameters are fixed, the noise MSE for the LS estimator is then determined by the ratio $K / A$. Thus, for example, if $A$ is doubled, the MSE performance can be maintained if $K$ is also doubled. This conclusion also holds for the MMSE estimator when the channels of different antennas are statistically identical.

\section{B. Leakage Effect for Non-Sample-Spaced Channels}

Here we investigate the leakage effect for the LS estimator. The analysis for the MMSE estimators is similar and omitted.

1) Expression of Leakage MSE: The MSE part caused by leakage is given by $E\left\{(\mathbf{B h})^{H}(\mathbf{B h})\right\}$. Let $b_{i, j}$ be the $(i, j)$ th element of $B$. In Appendix, we show that

$$
M S E_{\text {LS, leakage }}=\sum_{j=1}^{A L} \lambda_{j}^{2} \sum_{i=1}^{A N_{c}}\left\|b_{i, j}\right\|^{2},
$$

where $\lambda_{j}^{2}$ is the power of the $j$ th element in $\mathbf{h}$. We re-express $\mathbf{B}$ as

$$
\mathbf{B}=\left[\begin{array}{cccc}
\mathbf{B}_{1,1} & \mathbf{B}_{1,2} & \cdots & \mathbf{B}_{1, A} \\
\mathbf{B}_{2,1} & \mathbf{B}_{2,2} & \cdots & \mathbf{B}_{2, A} \\
\vdots & \vdots & \ddots & \vdots \\
\mathbf{B}_{A, 1} & \mathbf{B}_{A, 2} & \cdots & \mathbf{B}_{A, A}
\end{array}\right]
$$

where $\mathbf{B}_{i, j} \in \mathbb{C}^{N_{c} \times L}$. The $(k, n)$ th element of $\mathbf{B}_{i, i}$ is given by

$$
\begin{aligned}
B_{i, i}(k, n)=\frac{1}{\sqrt{N_{c}}} & \sum_{m=1}^{L^{\prime}} W^{k m} \frac{e^{j \pi\left(2 x_{1} \frac{K}{N_{c}}+K-1\right)\left(m-\tau_{i, n}\right) / K}}{K} \\
& \cdot \frac{\sin \left(\pi\left(m-\tau_{i, n}\right)\right)}{\sin \left(\frac{\pi}{K}\left(m-\tau_{i, n}\right)\right)}-\frac{1}{\sqrt{N_{c}}} W^{k \tau_{i, n}} \cdot
\end{aligned}
$$

The $(k, n)$ th element of $\mathbf{B}_{i, j}, i \neq j$ is given by

$$
\begin{aligned}
B_{i, j}(k, n)= & \frac{1}{\sqrt{N_{c}}} \sum_{m=1}^{L^{\prime}} W^{k m} \frac{\sin \left(\pi \cdot \theta_{i, j}(m, n)\right)}{\sin \left(\frac{\pi}{K} \theta_{i, j}(m, n)\right)} \\
& \times \frac{\exp \left(j \pi \cdot \theta_{i, j}(m, n) \cdot\left(2 x_{1} \frac{K}{N_{c}}+K-1\right) / K\right)}{K}
\end{aligned}
$$

where $\theta_{i, j}(m, n)=U(i-j)+\left(m-\tau_{j, n}\right)$.

2) Discussions: The matrix $\mathbf{B}$ characterizes the leakage effect. We have the following observations. In Fig. 2, we plot $\mathbf{h}$ and $\widehat{\mathbf{h}}_{L S}$ for a system with two transmit antennas. Each channel has six taps with an exponential power delay profile. Except for the second tap of Channel 1 which has $\tau_{1,2}=0.5$, all other tap delays are integers (from 0 to 5). As shown in Fig. 2, the power of Tap 2 of Antenna 1 also leaks to Antenna 2. In this experiment, $L^{\prime}$ is taken as 32 (maximal value for $L^{\prime}$ ) in order to show the whole time domain response. In Fig. 3(a), we plot the corresponding frequency domain response. The frequency domain response has severe fluctuations on the channel of Antenna 2, because the leakage from Antenna 1 has a strong presence at the high frequency end of Antenna 2. If $L^{\prime}$ is reduced to 16 (this can be viewed as time domain windowing), as shown

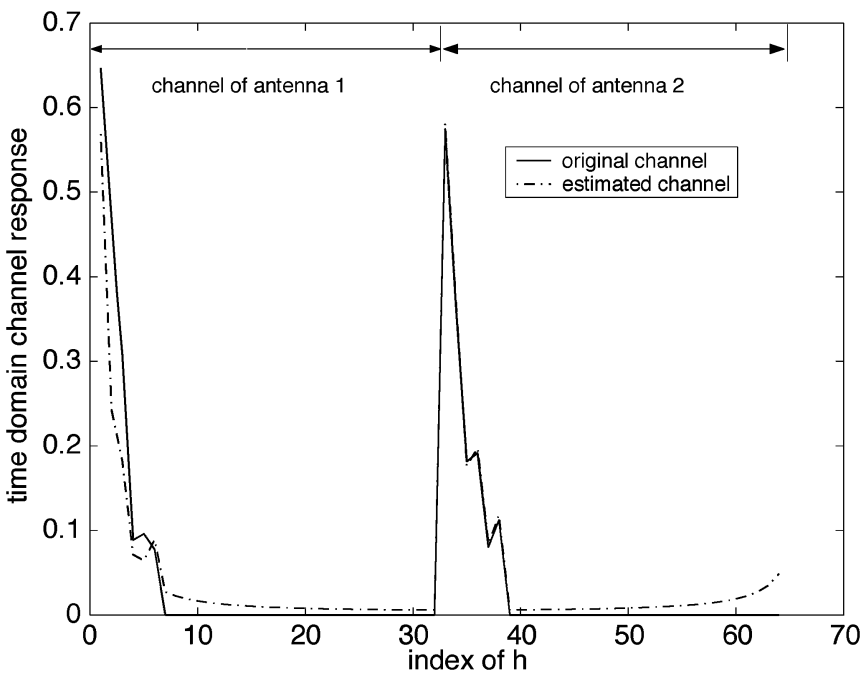

Fig. 2. Time domain channel responses.

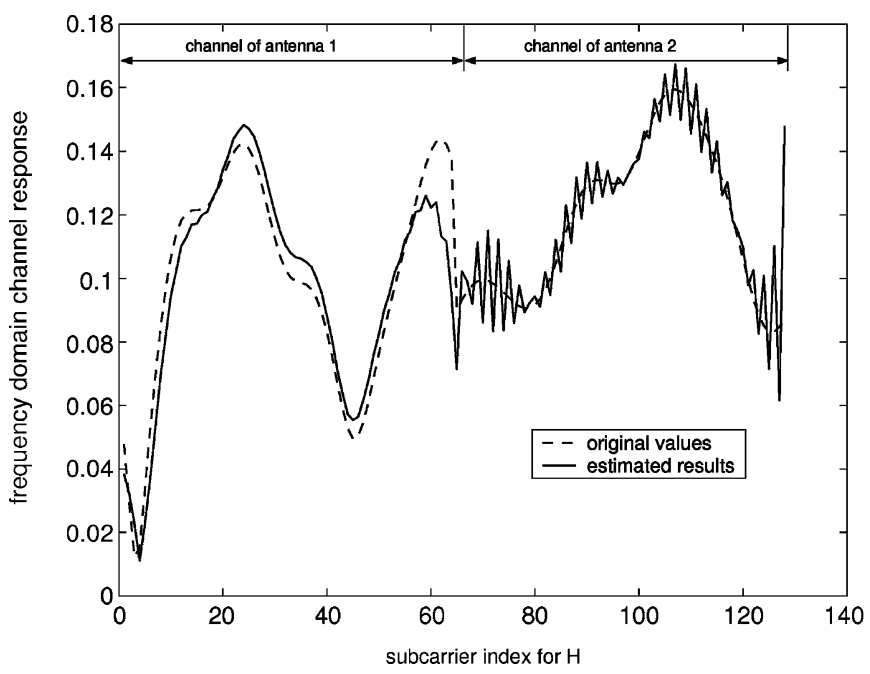

(a)

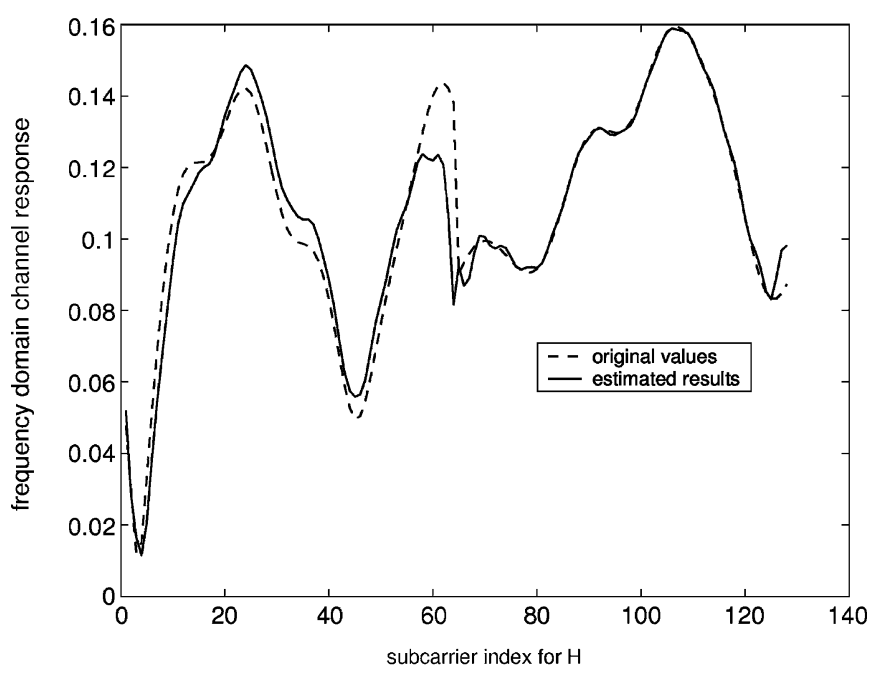

(b)

Fig. 3. Frequency domain channel responses. (a) $L^{\prime}=32$; (b) $L^{\prime}=16$.

in Fig. 3(b), the influence of leakage on the channel of Antenna 2 is almost removed. Correspondingly, the normalized MSE is 


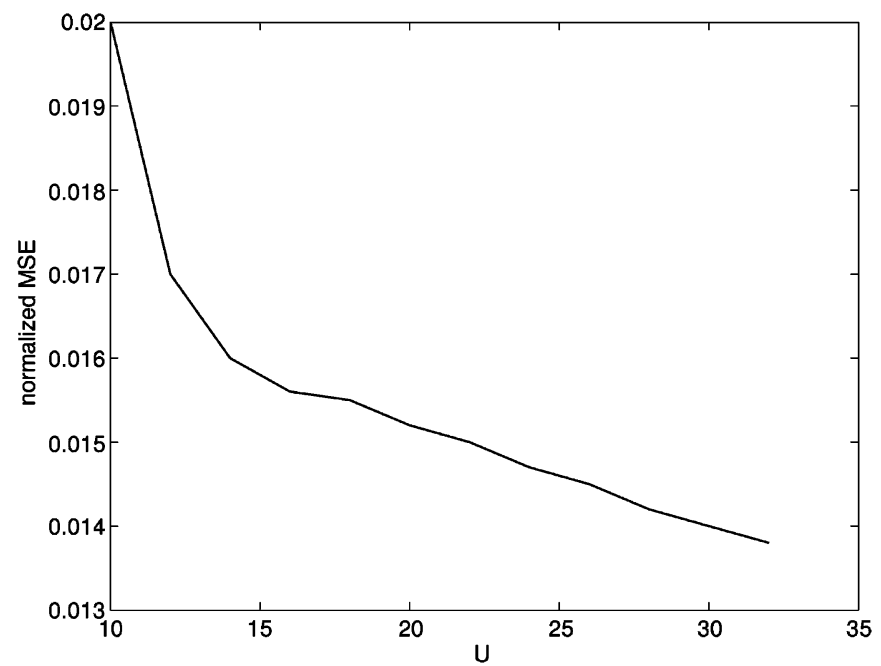

Fig. 4. Normalized MSE for different values of $U$.

reduced from 0.022 to 0.0132 . Here the normalized MSE is defined as $E\left\{\left(\widehat{\mathbf{H}}_{L S}-\mathbf{H}_{0}\right)^{H}\left(\widehat{\mathbf{H}}_{L S}-\mathbf{H}_{0}\right) / \mathbf{H}_{0}^{H} \mathbf{H}_{0}\right\}$.

Observation 1: Submatrix $\mathbf{B}_{i, j}$ represents the leakage from channel taps of antenna $j$ to those of antenna $i$. This indicates leakage exists not only among channel taps of the same antenna, but also across different antennas. On the other hand, a samplespaced channel tap produces no leakage to any other taps.

Observation 2: From (29), the amplitude of $B_{i, j}(m, n), i \neq$ $j$ is determined by

$$
\frac{\sin \left(\pi \cdot \theta_{i, j}(m, n)\right)}{K \sin \left(\frac{\pi}{K} \theta_{i, j}(m, n)\right)}
$$

where $\theta_{i, j}(m, n)=U(i-j)+\left(m-\tau_{i, n}\right)$. Obviously, a larger $U$ helps to reduce the amplitude of $B_{i, j}(m, n)$. Thus, to minimize the leakage effect across antennas, $U$ should be chosen as the largest possible value, which can be calculated as $\lfloor K / A\rfloor$. Here $\lfloor x\rfloor$ represents the largest integer that is smaller than $x$.

In Fig. 4, we plot the normalized MSE for different values of $U$. The channel is identical to the channel in Fig. 2 and $L^{\prime}$ is chosen to be 10. It is clear that the normalized MSE is steadily reduced with the increase of $U$.

Observation 3: When $U$ is chosen as $\rfloor K / A\rfloor$, the leakage effect is only related to the ratio $K / A$. Thus, the leakage effect can be alleviated by using more pilots, which directly leads to a larger $U$. For the same reason, given the number of pilots, less leakage is introduced if fewer channels are estimated simultaneously.

On the other hand, an increase in the number of antennas while maintaining the number of pilots should be equivalent to a proportional reduction in the number of pilots while keeping the number of antennas unchanged.

\section{Comparison Between Sample-Spaced and Non-Sample-Spaced Approaches}

The sample-spaced channel estimators can be simplified by orthogonal pilot sequences, but suffer from the leakage effect. In contrast, although better performance can be expected for the nonsample-spaced approach, much higher complexity is
TABLE I

ChANNEl DELAY PRofile (IN ns)

\begin{tabular}{|c|c|c|c|c|c|c|}
\hline Tap & 1 & 2 & 3 & 4 & 5 & 6 \\
\hline \hline Type 1 & 0 & 50 & 100 & 150 & 200 & 250 \\
\hline Type 2 & 0 & 60 & 100 & 140 & 180 & 220 \\
\hline Type 3 & 0 & 25 & 75 & 125 & 175 & 225 \\
\hline
\end{tabular}

involved. For nonsample-spaced approach, matrix inversion is needed. When the time delays of channel taps have to be estimated, as in [8], the inversion of matrix has to be computed on-line.

Obviously, the sample-spaced and nonsample-spaced approaches trade off complexity for performance.

\section{Simulation Results}

\section{A. Simulation Setup}

We simulate an OFDM system with 2 antennas for transmit diversity and 1 or 2 receive antennas. Alamouti's [17] spacetime block code (STBC) is adopted for performance evaluation. The parameters of the OFDM system are identical to those of IEEE 802.11a [19]. There are 64 subcarriers occupying $20 \mathrm{MHz}$ bandwidth at $5.4 \mathrm{GHz}$. Each OFDM symbol lasts for a duration of $4.0 \mu \mathrm{s}$, in which $0.8 \mu \mathrm{s}$ is the guard interval. The sampling period is $50 \mathrm{~ns}$. The two antennas have 6 taps of Rayleigh faded paths with the same exponential power delay profile. More specifically, we consider three types of channel delay profiles, as listed in Table I. Type 1 channel is a sample-spaced channel. Type 2 channel is nonsample-spaced with median leakage, and Type 3 channel is also nonsample-spaced with strong leakage, because the tap delays are in the middle of two sampling instances. The channel is quasistatic in the sense that it is constant in a block of two consecutive OFDM symbols (the block size for Alamouti's code) and varies independently from block to block. Orthogonal pilot sequences are employed, which are generated using the maximal possible value of $U$, as in (16).

In the simulation, the modulation scheme is the same for all data subcarriers with average symbol energy $E_{s}$, and the noise is complex additive white Gaussian with zero mean and variance $N_{0} / 2$ per dimension. The transmit power is split equally on two transmit antennas, so that the signal-to-noise ratio (SNR) is given as $E_{s} / N_{0}$.

\section{B. MSE of LS and MMSE Estimators}

In Fig. 6, we compare the normalized MSE of the LS and MMSE estimators for the three types of channels. The number of pilots is fixed at 64. The pilot SNR (PSNR) is the received SNR on the pilot subcarriers. From the results in Fig. 6, we observe that the MMSE estimator has better performance than the LS estimator at low PSNR, and almost the same performance at high PSNR. Most importantly, it is clear that the channel tap delay has a strong influence on the MSE performance. For the sample-spaced Type 1 channel, the MSE curve has a steady decay rate with respect to the increase of PSNR. However, the nonsample-spaced Type 2 and Type 3 channels all exhibit irreducible MSE floors. The MSE floor of Type 3 channel is more severe than that of Type 2 channel. Such MSE floors will lead to irreducible bit error rate (BER) floors. 


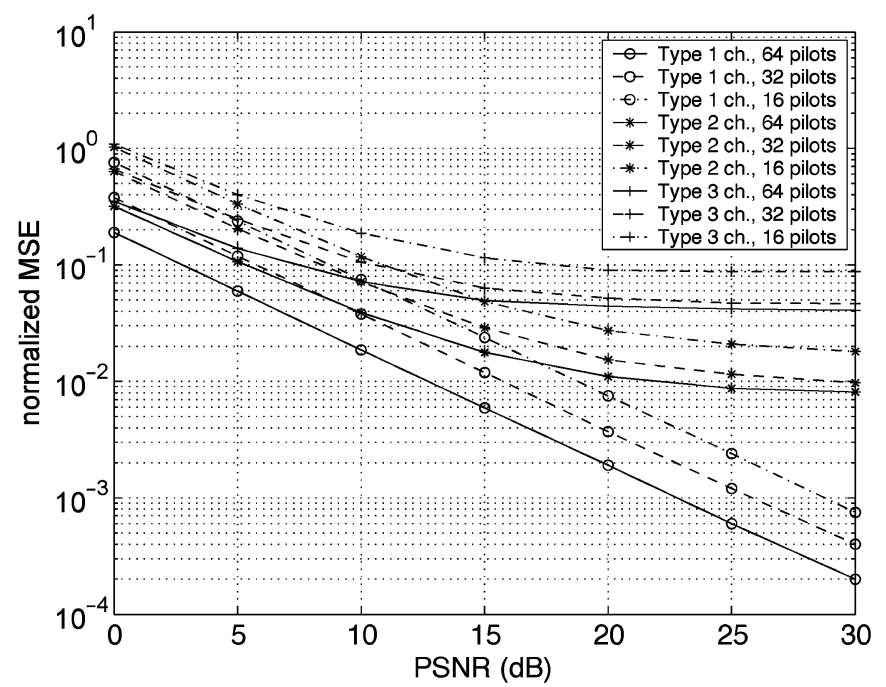

Fig. 5. Normalized MSE of LS estimator with different number of pilots.

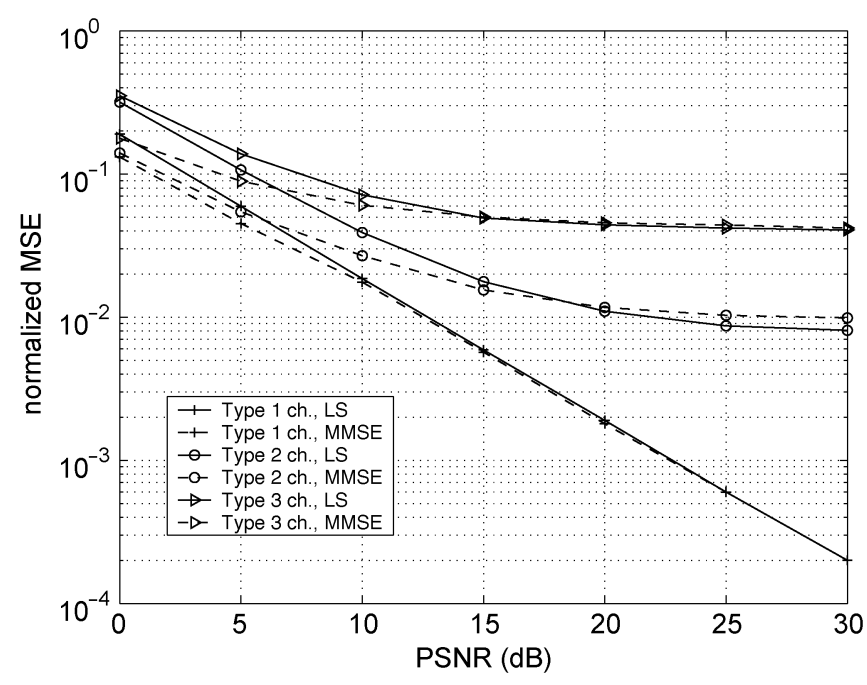

Fig. 6. MSE comparison for three different channels with 64 pilots.

\section{Influence of the Number of Pilots}

In Fig. 5, we present the normalized MSE of the LS estimators for the three types of channels with different number of pilots (see also Fig. 6). At the same time, the BER performance is presented in Fig. 7. Results for the MMSE estimator are not provided because they exhibit the same trend as the LS estimator. In Fig. 5, the MSE performance deteriorates as the number of pilots is reduced. For Type 1 channel, when the number of pilots is reduced, there is an almost constant PSNR gap between the two MSE curves. Thus, it is possible to maintain the MSE by increasing the PSNR to compensate for a reduction of pilots. Therefore, there is a tradeoff between PSNR and number of pilots for the sample-spaced channel, as already discussed in Section V-A-3. However, such a tradeoff does not exist for the nonsample-spaced Type 2 and Type 3 channels, because the MSE floor is higher when the number of pilots is reduced. In other words, for the nonsample-spaced channels, the MSE loss due to the reduction of pilots can not be compensated by the increase in PSNR.

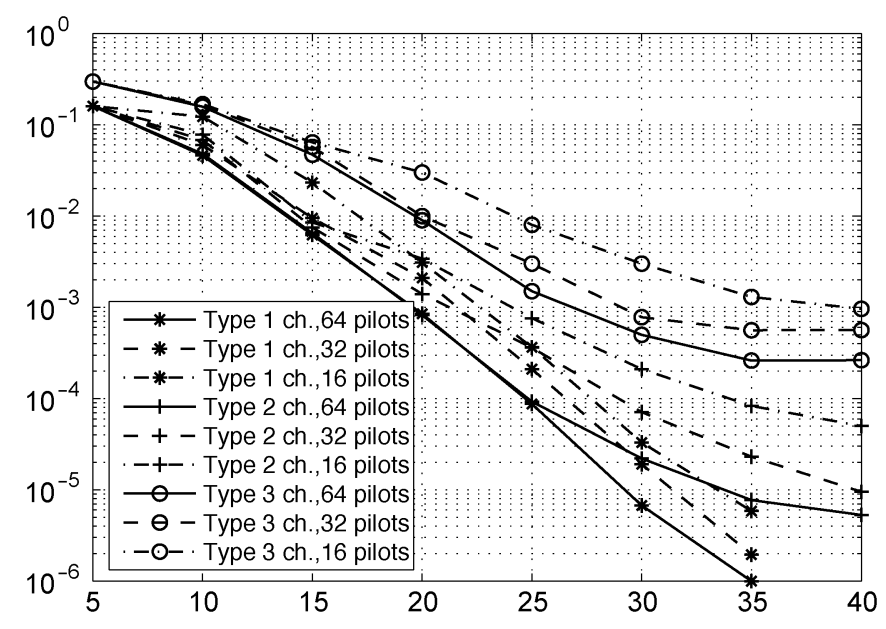

Fig. 7. BER performance of three channel types.

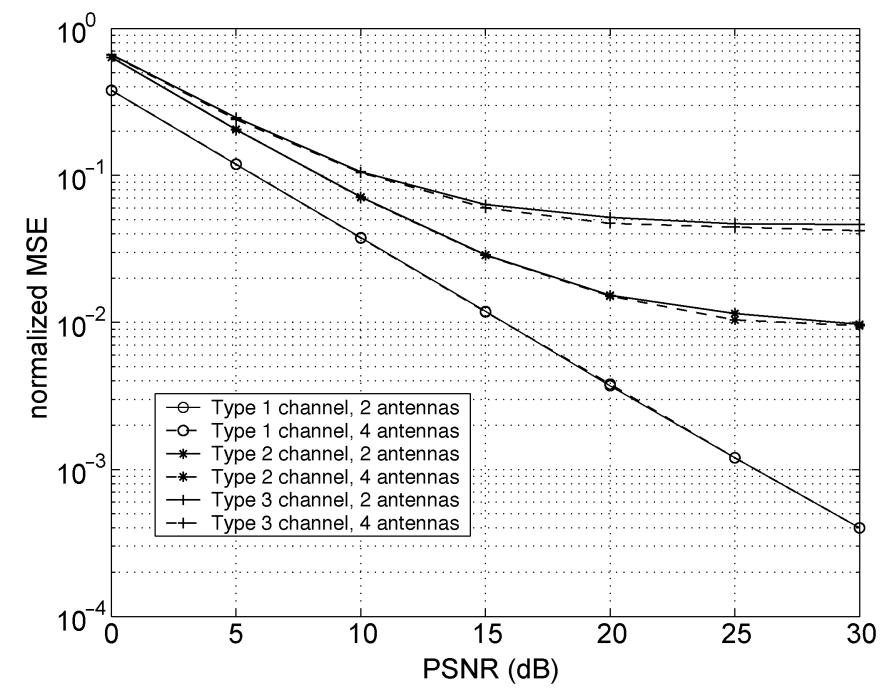

Fig. 8. Normalized MSE of LS estimator with different number of antennas.

\section{Influence of the Number of Antennas}

We investigate the influence of the number of antennas on the performance of the estimators. In Fig. 8, we plot the MSE performance of LS estimator when there are two and four antennas. For the two-antenna case, 32 pilots are used, and for the four-antenna case, 64 pilots are used. From the results in Fig. 8, we observe that for the same type of channel, the performance for having two antennas and 32 pilots is identical to that of having four antennas and 64 pilots. This phenomenon copes with the conclusion in Section V-B-2 that the performance is only determined by $U$, which is given by the ratio between the number of pilots and the number of antennas in our simulations.

\section{E. Performance With STBC}

We study the BER performance of a space-time block coded OFDM systems using Alamouti's scheme [17]. We adopt the LS estimator and employ 64 pilots. The MMSE estimator is not used because it has similar performance as the LS estimator at high PSNR and it involves higher complexity. In the experiment, 


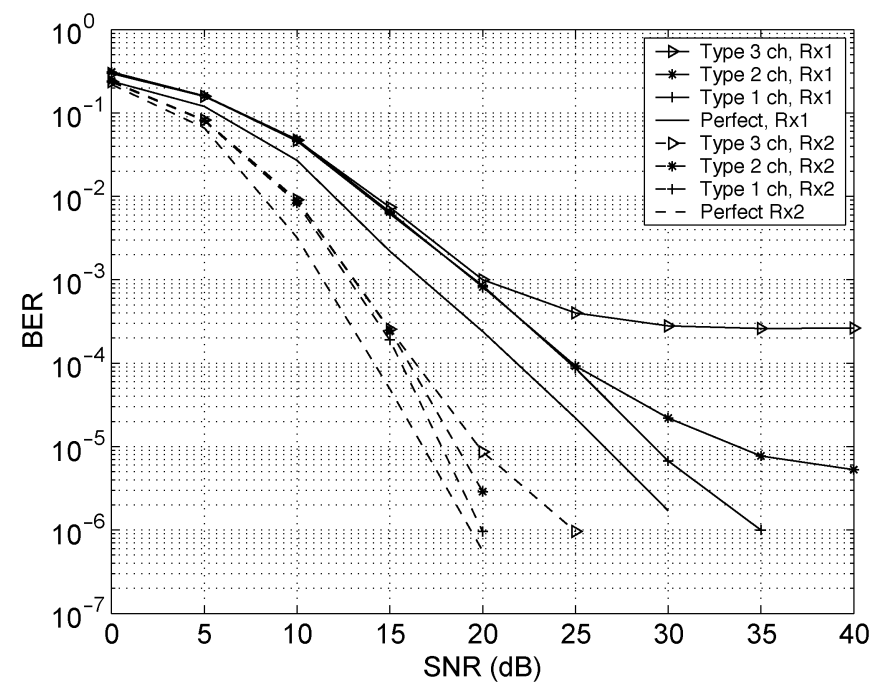

Fig. 9. BER performance of a space-time coded OFDM system with LS channel estimator for one and two receive antennas.

the six subcarriers on each side of the spectrum boundary are not used for carrying data.

In Fig. 9, we present BER results for the three types of channels with QPSK modulation. The results with perfect channel knowledge are also given for comparison. In the experiment, we set PSNR the same as SNR on data subcarriers. Based on the results in Fig. 9, we find that with one receive antenna, there is a BER floor for both Type 2 and Type 3 channels. With two receive antennas, BER floor is still observable for Type 3 channel, but does not appear for the Type 2 channel. Furthermore, BER for all the types of channels can reach $10^{-6}$. This shows the STBC code is less sensitive to channel estimation error with more receive diversity.

\section{CONCLUSION}

In this paper, we have presented pilot-assisted LS and MMSE channel estimators for OFDM systems with transmit diversity. Furthermore, orthogonal pilot sequences are designed so that the complexity of the estimator is reduced. Performance analysis is conducted on the estimators for both sample-spaced and nonsample-spaced channels. The MSE can be viewed as having two parts: the noise part and the leakage part. For the noise part, estimator performance is only determined by the total pilot energy. For the leakage part, the influence of leakage is mainly determined by the ratio between the number of pilots and number of antenna channels estimated simultaneously. Thus, leakage can be reduced by using more pilots, or estimating fewer channels simultaneously. Simulation results have shown that the leakage effect of the nonsample-spaced channel leads to irreducible BER floors, which can be mitigated with more receive diversity.

APPENDIX

Note

$$
\begin{aligned}
\mathbf{P} & =\mathbf{S W} \\
& =\left[\mathbf{S}_{1} \mathbf{W}_{1} \mathbf{S}_{2} \mathbf{W}_{2} \cdots \mathbf{S}_{A} \mathbf{W}_{A}\right]
\end{aligned}
$$

Then $\mathbf{Q}=\mathbf{P}^{H} \mathbf{P}$ is expressed as

$$
\begin{aligned}
\mathbf{Q} & =\left[\mathbf{S}_{1} \mathbf{W}_{1} \mathbf{S}_{2} \mathbf{W}_{2} \cdots \mathbf{S}_{A} \mathbf{W}_{A}\right]^{H}\left[\mathbf{S}_{1} \mathbf{W}_{1} \mathbf{S}_{2} \mathbf{W}_{2} \cdots \mathbf{S}_{A} \mathbf{W}_{A}\right] \\
& =\left[\begin{array}{cccc}
\mathbf{Q}_{1,1} & \mathbf{Q}_{1,2} & \cdots & \mathbf{Q}_{1, A} \\
\mathbf{Q}_{2,1} & \mathbf{Q}_{2,2} & \cdots & \mathbf{Q}_{2, A} \\
\vdots & \vdots & \ddots & \vdots \\
\mathbf{Q}_{A, 1} & \mathbf{Q}_{A, 2} & \cdots & \mathbf{Q}_{A, A}
\end{array}\right]
\end{aligned}
$$

where $\mathbf{Q}_{i, j}=\mathbf{W}_{i}^{H} \mathbf{S}_{i}^{H} \mathbf{S}_{j} \mathbf{W}_{j}$, and $\mathbf{Q}_{i, j} \in \mathbb{C}^{L^{\prime} \times L^{\prime}}$. Let $Q_{i, j}(m, n)$ denote the element on the $m$ th row and $n$th column of matrix $\mathbf{Q}_{i, j}$. We have

$$
Q_{i, j}(m, n)=\sum_{k=1}^{K} s_{i}^{H}\left(x_{k}\right) s_{j}\left(x_{k}\right) W^{-x_{k} m} W^{x_{k} n} .
$$

Note in (30) that $x_{k}$ corresponds to the subcarrier location of the $k$ th pilot.

We can show that by proper selection of pilot sequences, the submatrices $\mathbf{Q}_{i, j}$ of $\mathbf{Q}$ can be simplified to

$$
\mathbf{Q}_{i, j}= \begin{cases}\frac{K}{N_{c}} E_{0} \mathbf{I}_{L^{\prime}} & i=j \\ \mathbf{0} & i \neq j\end{cases}
$$

where $\mathbf{I}_{L^{\prime}}$ is an identity matrix of dimension $L^{\prime}, \mathbf{0}$ is a zero matrix of size $L^{\prime} \times L^{\prime}$, and $E_{0}$ is the pilot energy.

First, we demonstrate that the diagonal submatrices can be identity matrices with constant modulus modulation on pilot subcarriers and proper selection of pilot locations. Let $\left|s_{i}\left(x_{k}\right)\right|^{2}=E_{0}$. From (30), we have

$$
\begin{aligned}
Q_{i, i}(m, n) & =\sum_{k=1}^{K} s_{i}^{H}\left(x_{k}\right) s_{i}\left(x_{k}\right) W^{-x_{k} m} W^{x_{k} n} \\
& =\frac{1}{N_{c}} \sum_{k=1}^{K}\left|s_{i}\left(x_{k}\right)\right|^{2} e^{-j 2 \pi x_{k}(n-m) / N_{c}} \\
& =\frac{1}{N_{c}} E_{0} \sum_{k=1}^{K} e^{-j 2 \pi x_{k}(n-m) / N_{c}} .
\end{aligned}
$$

Obviously, $Q_{i, i}(n, n)=\left(K / N_{c}\right) E_{i}$. When $m \neq n$, the sum depends on pilot locations $x_{k}, 1 \leq k \leq K$. The sum in (32) is zero for $m \neq n$ when the following conditions are satisfied: 1) $N_{c} / K$ is an integer, 2) the pilot locations are equally spaced. We give the proof as follows.

Let $D=N_{c} / K$. The $k$ th pilot location can be generally expressed as

$$
x_{k}=x_{1}+(k-1) D
$$

for $2 \leq k \leq K$. Then (32) can be re-formulated as

$$
\begin{aligned}
Q_{i, i}(m, n)= & \frac{1}{N_{c}} E_{0} \sum_{k=1}^{K} e^{-j 2 \pi\left(x_{1}+(k-1) D\right)(n-m)} \\
= & \frac{1}{N_{c}} E_{0} e^{-j 2 \pi\left(x_{1}-D\right)(n-m) / N_{c}} \\
& \times \sum_{k=1}^{K} e^{-j 2 \pi k D(n-m) / N_{c}} \\
= & q \sum_{k=1}^{K} e^{-j 2 \pi k(n-m) N_{c} / K / N_{c}}
\end{aligned}
$$


where $q=\left(K E_{0} / N_{c}\right) e^{-j 2 \pi\left(x_{1}-D\right)(n-m)}$. Then it is obvious that $Q_{i, i}(m, n)=0, m \neq n$. Finally, we get

$$
\mathbf{Q}_{i, i}=\frac{K}{N_{c}} E_{o}, \quad 1 \leq i \leq A .
$$

Given the above conditions for pilot locations, the pilot sequences can be further designed to make the off-diagonal submatrices zero matrices. We set

$$
s_{i}\left(x_{k}\right)=s_{1}\left(x_{k}\right) e^{-j 2 \pi U(i-1) x_{k} / N_{c}}
$$

for $i=2, \ldots, A$. Then from (30), we have

$$
\begin{aligned}
Q_{i, j}(m, n)= & \sum_{k=1}^{K} s_{1}^{H}\left(x_{k}\right) e^{j 2 \pi U(i-1) x_{k} / N_{c}} W^{-x_{k} m} \\
& \times s_{1}\left(x_{k}\right) e^{-j 2 \pi U(j-1) x_{k} / N_{c}} W^{x_{k} n} \\
= & \frac{E_{0}}{N_{c}} \sum_{k=1}^{K} e^{j 2 \pi[U(i-j)-(n-m)] x_{k} / N_{c}}
\end{aligned}
$$

According to the previous design of pilot locations, the summation in (36) is just

$$
Q_{i, j}(m, n)=\frac{E_{0}}{N_{c}} \delta(U(i-j)-(n-m))
$$

It is clear that $\mathbf{Q}_{i, j}$ is a zero matrix if and only if $U(i-j)-$ $(m-n) \neq 0$. When $i \neq j$, there is $|U(i-j)|>U$ for all $i, j$. Since $|m-n| \leq L^{\prime}-1$, there is always $|m-n|<|U(i-j)|$ as long as $U \geq L^{\prime}$. Thus $U(i-j)-(m-n)$ is always nonzero for $i \neq j$, and $\mathbf{Q}_{i, j}$ is a zero matrix. Further, it is obvious that $A$ orthogonal sequences can always be obtained as long as

$$
A U \leq K .
$$

With the above design of pilot sequences, $\mathbf{Q}$ becomes

$$
\mathbf{Q}=\frac{K E_{0}}{N_{c}}\left[\begin{array}{cccc}
\mathbf{I}_{L^{\prime}} & \mathbf{0} & \cdots & \mathbf{0} \\
\mathbf{0} & \mathbf{I}_{L^{\prime}} & \cdots & \mathbf{0} \\
\vdots & \vdots & \ddots & \vdots \\
\mathbf{0} & \mathbf{0} & \cdots & \mathbf{I}_{L^{\prime}}
\end{array}\right]
$$

From (21),

$$
\begin{aligned}
M S E_{\text {LS, leakage }} & =E\left\{(\mathbf{B h})^{H}(\mathbf{B h})\right\} \\
& =E\left\{\operatorname{tr}\left((\mathbf{B h})(\mathbf{B h})^{H}\right)\right\} \\
& =\operatorname{tr}\left(\mathbf{B} E\left\{\mathbf{h} \mathbf{h}^{H}\right\} \mathbf{B}^{H}\right) \\
& =\operatorname{tr}\left(\mathbf{B} \mathbf{R}_{h h} \mathbf{B}^{H}\right) .
\end{aligned}
$$

The form of $\mathbf{R}_{h h}$ has been given in (14). We now re-express $\mathbf{R}_{h h}=\operatorname{diag}\left\{\lambda_{1}^{2},, \lambda_{2}^{2}, \cdots, \lambda_{A L}^{2}\right\}$, where $\lambda_{j}^{2}$ is the power of the $j$ th element in vector $\mathbf{h}$. Let $\mathbf{B}=\left[\mathbf{B}_{1} \mathbf{B}_{2} \cdots \mathbf{B}_{A L}\right]$, where
$\mathbf{B}_{j}=\left[b_{1, j} b_{2, j} \cdots b_{A N_{c}, j}\right]^{T}, j=1, \ldots, A L$ and $b_{i, j}$ is the $(i, j)$ th element of $\mathbf{B}$. Then

$$
\begin{aligned}
\operatorname{tr}\left(\mathbf{B R}_{h h} \mathbf{B}^{H}\right) & =\operatorname{tr}\left(\sum_{j=1}^{A L} \lambda_{j}^{2} \mathbf{B}_{j} \mathbf{B}_{j}^{H}\right) \\
& =\sum_{j=1}^{A L} \lambda_{j}^{2} \operatorname{tr}\left(\mathbf{B}_{j} \mathbf{B}_{j}^{H}\right) \\
& =\sum_{j=1}^{A L} \lambda_{j}^{2} \mathbf{B}_{j}^{H} \mathbf{B}_{j} \\
& =\sum_{j=1}^{A L} \lambda_{j}^{2} \sum_{i=1}^{A N_{c}}\left\|b_{i, j}\right\|^{2} .
\end{aligned}
$$

Let $\mathbf{Q}^{\prime}=\left(\mathbf{P}^{H} \mathbf{P}\right)^{-1} \mathbf{P}^{H} \mathbf{P}_{N S} \in \mathbb{C}^{A L^{\prime} \times A L}$. There is

$$
\mathbf{Q}^{\prime}=\left[\begin{array}{cccc}
\mathbf{Q}_{1,1}^{\prime} & \mathbf{Q}_{i, 2}^{\prime} & \cdots & \mathbf{Q}_{1, A}^{\prime} \\
\mathbf{Q}_{2,1}^{\prime} & \mathbf{Q}_{2,2}^{\prime} & \cdots & \mathbf{Q}_{2, A}^{\prime} \\
\vdots & \vdots & \ddots & \vdots \\
\mathbf{Q}_{A, 1}^{\prime} & \mathbf{Q}_{A, 2}^{\prime} & \cdots & \mathbf{Q}_{A, A}^{\prime}
\end{array}\right]
$$

where $\mathbf{Q}_{i, j}^{\prime} \in \mathbb{C}^{L^{\prime} \times L}$ and the $(m, n)$ th element of $\mathbf{Q}_{i, j}^{\prime}$ is given by

$$
\begin{aligned}
Q_{i, j}^{\prime}(m, n)= & \frac{e^{j \varphi_{i, j}(m, n)}}{K} \sum_{k=1}^{K} \exp \left(j 2 \pi \cdot \theta_{i, j}(m, n) \cdot k / K\right) \\
= & \frac{e^{j \varphi_{i, j}(m, n)}}{K} \exp \left(j \pi \cdot \theta_{i, j}(m, n) \cdot(K+1) / K\right) \\
& \times \frac{\sin \left(\pi \cdot \theta_{i, j}(m, n)\right)}{\sin \left(\frac{\pi}{K} \theta_{i, j}(m, n)\right)} \\
= & \frac{\exp \left(j \pi \cdot \theta_{i, j}(m, n) \cdot\left(2 x_{1} \frac{K}{N_{c}}+K-1\right) / K\right)}{K} \\
& \times \frac{\sin \left(\pi \cdot \theta_{i, j}(m, n)\right)}{\sin \left(\frac{\pi}{K} \theta_{i, j}(m, n)\right)}
\end{aligned}
$$

where $\theta_{i, j}(m, n)=U(i-j)+\left(m-\tau_{j, n}\right)$ and $\varphi_{i, j}(m, n)=$ $2 \pi \theta_{i, j}(m, n)\left(x_{1}-\left(N_{c} / K\right)\right) / N_{c}$ Finally, it is straightforward to obtain (28) and (29).

\section{REFERENCES}

[1] R. Blum, Y. Li, J. Winters, and Q. Yan, "Improved space-time coding for MIMO-OFDM wireless communications," IEEE Trans. Commun., vol. 49, no. 11, pp. 1873-1878, Nov. 2001.

[2] J. J. van de Beek, O. Edfors, M. Sandell, S. K. Wilson, and P. O. Borjesson, "On channel estimation in OFDM systems," in IEEE Proc. Vehic. Technol. Conf., 1995, pp. 815-819.

[3] C. Yeh, Y. Lin, and Y. Wu, "OFDM system channel estimation using time-domain training sequence for mobile reception of digital terrestria broadcasting," IEEE Trans. on Broadcasting, vol. 46, no. 2, Sep. 2000.

[4] H. Minn, "An investigation into time-domain approach for OFDM channel estimation," IEEE Trans. on Broadcasting, vol. 46, no. 4, Dec. 2000.

[5] O. Edfors et al., "OFDM channel estimation by singular value decomposition," IEEE Trans. Commun., vol. 46, no. 7, pp. 931-939, Jul. 1998

[6] Y. Li, L. J. Cimini Jr., and N. R. Sollenberger, "Robust channel estimation for OFDM systems with rapid dispersive fading channels," IEEE Trans. Commun., vol. 46, no. 7, pp. 902-914, Jul. 1998.

[7] Y. Li, "Pilot-symbol-aided channel estimation for OFDM in wireless systems," IEEE Trans. Veh. Technol., vol. 49, no. 4, pp. 1207-1215, Jul. 2000. 
[8] B. Yang, K. B. Letaief, R. S. Cheng, and Z. Cao, "Channel estimation for OFDM transmission in multipath fading channels based on parametric channel modeling," IEEE Trans. Commun., vol. 49, no. 3, pp. 467-479, Mar. 2001.

[9] B. Yang, Z. Cao, and K. B. Letaief, "Analysis of low-complexity windowed DFT-based MMSE channel estimator for OFDM systems," IEEE Trans. Commun., vol. 49, no. 11, pp. 1977-1987, Nov. 2001.

[10] M. Morelli and U. Mengali, "A comparison of pilot-aided channel estimation methods for OFDM systems," IEEE Trans. Signal Processing, vol. 49, no. 12, pp. 3065-3073, Dec. 2001.

[11] Y. Li, N. Seshadri, and S. Ariyavisitakul, "Channel estimation for OFDM systems with transmitter diversity in mobile wireless channels," IEEE J. Select. Areas Commun., vol. 17, no. 3, pp. 461-471, Mar. 1999.

[12] Y. Li, "Simplified channel estimation for OFDM systems with multiple transmit antennas," IEEE Trans. Wireless Commun., vol. 1, no. 1, pp. 67-75, Jan. 2002.

[13] I. Barhumi, G. Leus, and M. Moonen, "Optimal training design for MIMO OFDM systems in mobile wireless channels," IEEE Trans. Signal Processing, vol. 51, no. 6, pp. 1615-1624, Jun. 2003.

[14] H. Minn, D. Kim, and V. K. Bhargava, "A reduced complexity channel estimation for OFDM systems with transmit diversity in mobile wireless channels," IEEE Trans. Commun., vol. 50, no. 5, pp. 799-807, May 2002.

[15] R. Chen and K. B. Letaief, "Channel estimation for space time coded OFDM systems in nonsample-spaced multipath channels," in IEEE WCNC'2002, vol. 1, Mar. 2002, pp. 61-66.

[16] S. M. Kay, Fundamentals of Statistical Signal Processing: Estimation Theory: Prentice Hall, 1993.

[17] S. Alamouti, "A simple transmit diversity technique for wireless communications," IEEE J. Select. Areas Commun., vol. 16, no. 8, pp. 1451-1458, Oct. 1998.

[18] V. Tarokh, H. Jafarkhani, and A. Calderbank, "Space-time block codes from orthogonal designs," IEEE Trans. Inform. Theory, vol. 45, no. 5, pp. 1456-1467, Jul. 1999.

[19] Wireless LAN Medium Access Control (MAC) and Physical Layer (PHY) specification: High-speed Physical Layer in the $5 \mathrm{GHz}$ Band, IEEE std 802.11a-1999.

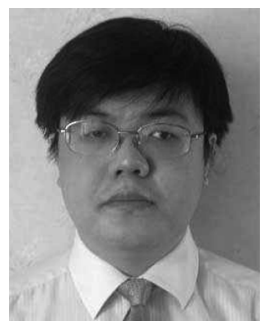

Dongxu Shen (S'97-M'01) received the B.S. degree from the Harbin University of Engineering, Harbin, China, in 1995, and the M.S. and Ph.D. degrees from Rensselear Polytechnic Institute, Troy, NY, in 1999 and 2001, respectively, all in electrical engineering.

From 2001 to 2004, he was a Research Associate at the Department of Electrical and Electronic Engineering, University of Hong Kong, Hong Kong. His research interests include smart antenna, OFDM systems, media access, scheduling, and cross-layer optimization.

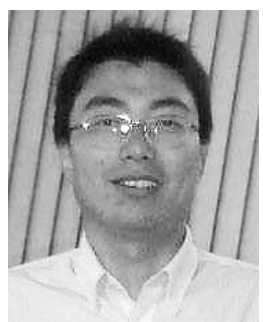

Zhifeng Diao (S'04) received the B.S. and M.S. degrees from Xidian University, Xi'an, China, in 1999 and 2002, respectively. $\mathrm{He}$ is currently working toward the Ph.D degree at the Department of Electrical and Electronic Engineering, University of Hong Kong, Hong Kong, China.

His research interests include wireless scheduling algorithm design, OFDM, MC-CDMA, cross-layer design, and smart antennas.

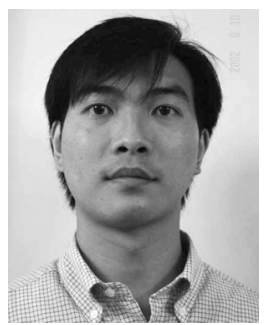

Kai-Kit Wong (M’01) received the B.Eng, M.Phil, and Ph.D. degrees in electrical and electronic engineering from the Hong Kong University of Science and Technology, Hong Kong, in 1996, 1998, and 2001, respectively.

After graduation, he joined the Department of Electrical and Electronic Engineering, University of Hong Kong, as a Research Assistant Professor for three years. From January to June 2004, he was with the Smart Antennas Research Group of Stanford University as a Visiting Assistant Professor. Before that, he spent a half-year at the Wireless Communications Research Department of Lucent Technologies, Bell-Labs, Holmdel, as a Visiting Research Scholar. In 2005, he joined the Department of Engineering, University of Hull, as a Lecturer. He has worked in several areas including smart antennas, space-time processing/coding, and equalization. His current research interests center around the joint optimization of smart antennas for multiuser wireless communication systems.

Dr. Wong was a corecipient of IEEE Vehicular Technology Society (VTS) Japan Chapter Award of the VTC2000-Spring in Japan.

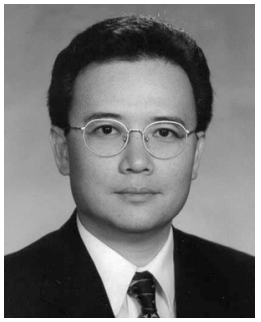

Victor O. K. Li (S'80-M'81-SM'86-F'92) (http:// www.eee.hku.hk/staff_personal/vli.htm) received the S.B., S.M., E.E., and Sc.D. degrees in electrical engineering and computer science from the Massachusetts Institute of Technology, Cambridge, in 1977, 1979, 1980, and 1981, respectively.

He joined the University of Southern California (USC), Los Angeles, CA, in February 1981 and became a Professor of electrical engineering and the $\mathrm{Di}$ rector of the USC Communication Sciences Institute. Since September 1997, he has been with the University of Hong Kong, Hong Kong, where he is a Chair Professor of information engineering at the Department of Electrical and Electronic Engineering. He has also served as the Managing Director of Versitech Ltd. (http://www.versitech.com.hk/), the technology transfer and commercial arm of the University, and on various corporate boards. His research is in information technology, including all-optical networks, wireless networks, and Internet technologies and applications.

Dr. Li is a Principal Investigator of the Area of Excellence in Information Technology funded by the Hong Kong government. Sought by government, industry, and academic organizations, he has lectured and consulted extensively around the world. He has been appointed to the Hong Kong Information Infrastructure Advisory Committee by the Chief Executive of the Hong Kong Special Administrative Region. He also serves on the Innovation and Technology Fund (Electronics) Vetting Committee, the Small Entrepreneur Research Assistance Program Committee, the Engineering Panel of the Research Grants Council, and the Task Force for the Hong Kong Academic and Research Network (HARNET) Development Fund of the University Grants Committee. He chaired the Computer Communications Technical Committee of the IEEE Communications Society from 1987 to 1989 and the Los Angeles Chapter of the IEEE Information Theory Group from 1983 to 1985 . He cofounded the International Conference on Computer Communications and Networks (IC3N) and chaired its Steering Committee from 1992 to 1997. He also chaired various international workshops and conferences, including, most recently, IEEE INFOCOM 2004. He has served as an Editor of IEEE Network, IEEE JSAC Wireless Communications Series, and Telecommunication Systems. He also guest edited Special Issues of IEEE JSAC, Computer Networks and ISDN Systems, and KICSIEEE Journal of Communications and Networking. He is now serving as an Editor of ACM Kluwer Wireless Networks and IEEE Communications Surveys and Tutorials. He was a Distinguished Lecturer at the University of California at San Diego, at the National Science Council of Taiwan, and at the California Polytechnic Institute. He has also delivered keynote speeches at many international conferences. He has received numerous awards, including, most recently, the U.K. Royal Academy of Engineering Senior Visiting Fellowship in Communications, the Outstanding Researcher Award of the University of Hong Kong, the Croucher Foundation Senior Research Fellowship, and the Bronze Bauhinia Star, Government of the Hong Kong Special Administrative Region, China. He is a Fellow of the HKIE and the IAE. 